\title{
An Improved Delay-Dependent Globally Asymptotically Stability Criterion for the Static Neural Networks with Time-Varying Delay
}

\author{
Kai Mao \\ Institute of Systems Science and Mathematics, \\ Department of basic sciences, \\ Naval Aeronautical and astronautical university \\ Yantai, Shandong, 264001, P R China
}

\author{
Bao Shi \\ Department of basic sciences, \\ Naval Aeronautical and Astronautical university \\ Yantai, Shandong, 264001, P R China
}

\author{
Shudong Zhang \\ Institute of Systems Science and Mathematics, \\ Department of basic sciences, \\ Naval Aeronautical and astronautical university \\ Yantai, Shandong, 264001, P R China
}

\begin{abstract}
The globally asymptotic stability for static neural networks with time-varying delay is concerned in this paper. By delay fractioning technique and taking more delayed-state variables into account, a newly Lyapunov-Krasovskii Functional was constructed, together with the Jessen integral inequality and convex combination method, a delay-dependent global stability criterion is obtained, it is less conservative than some existing ones. Example is provided to show the effectiveness and reduced conservatism of the proposed results.
\end{abstract}

Keywords-static neural networks; lyapunov-krasovskii functional; delay fractioning method; convex combination method; LMIs

\section{INTRODUCTION}

In the past few decades, recurrent neural networks (RNNs) have found extensive investigation due to its applications in a wide range of fields such as pattern recognition, signal processing, combinational optimization, associative memory, etc [1]. when RNNs are used to solve a certain problem,

As mentioned above, the existence, uniqueness and globally stability of its equilibrium point is of great both theoretical and practical importance. There have been many interesting results on global stability in the literature [2-22], and the references cited therein.On one hand, in electric implementation, time delays are often inevitable due to the finite switching speed of amplifiers or finite information processing speed, and they are often a resource of instability or oscillation. On the other hand, some neural networks with time delays do not have the dynamical behavior we need. To solve this problem, there exists one way that we can bring suitable time delays into neural networks without time delay, Yang and Cao have employed delayed projection neural network to solve quadratic programming[23]. Li proposed a delayed neural network to solve convex programming problems and pointed out that one can achieve the exact optimal solutions by choosing suitable delays [24]. Therefore, the stability of neural networks with time delay is of great importance both in theory and practice. A great number of stability results on neural networks with time delay have been proposed [4-8,12-22].

RNNs can be classified into the typical static neural networks and local field neural networks, according to whether the neuron states(the external states of neurons)or local field states(the internal states of neurons)are taken as basic variables[2]. For local field neural networks, many interesting results on stability analysis have been derived extensively. For example, the delay-dependent stability result of the neural networks with a constant delay can be found in[16-18], while the case of time-varying delays ones were reported in[15,19,20]. As far as distributed delays were considered, the stability results, can see $[21,22]$ and references therein.

Though the local neural networks have been investigated extensively, to the best of our knowledge, the static neural networks have been received relatively little attention, with only a few results available. In [4], the authors have studied the static neural networks for nonlinear optimization with a continuously differentiable objective function and bound constraints. A precise theoretical comparative study on the dynamics of local field neural networks and static neural networks was conducted in [3]. Some sufficient conditions for the globally asymptotically stability (GAS) and globally exponentially stability (GES) of the static neural networks without time delay were given in [5], and the robust GES analysis for interval static neural networks via linear matrix inequality (LMI) approaches was proposed in [6]. As far as the static neural networks with constant delay, the delayindependent GAS and GES conditions were developed in [7] and [10], respectively. By introducing the free-weighting matrices and through the use of Newton-Leibnitz formula to estimate elaborately the derivative of the constructed Lyapunov functional, a delay-dependent GAS result was obtained in[8]. 
For the static neural networks with time-varying delay, in [9], the Jensen integral inequality was used to estimate the derivative of the Lyapunov functional without introducing a free-weighting matrix, a delay-dependent GAS result was derived.

Inspired by the fact that "the static neural networks have been received relatively little attention", in this paper, based on a newly constructed Lyapunov-Krasovskii functional, the delay fractioning method and the Jensen integral inequality were used to estimate the upper bound of the derivative of the Lyapunov functional, a less conservative delay-dependent GAS criterion was developed in form of LMIs, together with the convex combination method.

\section{PROBLEM FORMULATION}

We consider the following static recurrent neural networks with time-varying delays:

$$
\begin{aligned}
& \dot{u}_{i}(t)=-a_{i} u_{i}(t)+g_{i}\left(\sum_{j=1}^{n} w_{i j} u_{j}(t-\tau(t))+J_{i}\right), i=1,2, \cdots, n, \\
& u(t)=\psi(t),-\tau \leq t \leq 0 .
\end{aligned}
$$

Or equivalently, in the matrix-vector form

$$
\begin{aligned}
& \dot{u}(t)=-A u(t)+g(W u(t-\tau(t))+J), \\
& u(t)=\psi(t),-\tau \leq t \leq 0 .
\end{aligned}
$$

where $u(t)=\left(u_{1}(t), u_{2}(t), \cdots, u_{n}(t)\right)^{T}$ is the state vector associated with the $n$ neurons; $g(u(t))=\left(g_{1}\left(u_{1}(t)\right), g_{2}\left(u_{2}(t)\right), \cdots, g_{n}\left(u_{n}(t)\right)\right)^{T}$ represents the neuron activation functions; $\boldsymbol{A}=\operatorname{diag}\left(a_{1}, a_{2}, \cdots, a_{n}\right)>\mathbf{0} ; \boldsymbol{W}=\left(w_{i j}\right)_{n \times n}$ is the delayed connection weight matrix; $J=\left(J_{1}, J_{2}, \cdots, J_{n}\right)^{T}$ is the constant input vector from outside the system; $\tau(t)$ is the time delay satisfying $0 \leq \tau(t) \leq \tau$ and $\dot{\tau}(t) \leq \mu ; \psi(t),-\tau \leq t \leq 0$ is the initial condition of system(1)

To obtain our results, the following assumption will be made throughout this paper:

Assumption The activation function satisfies the following condition

$$
l_{i}^{-} \leq \frac{g_{i}(x)-g_{i}(y)}{x-y} \leq l_{i}^{+}, \forall x, y \in R, x \neq y, i=1,2, \cdots, n
$$

where $l_{i}^{-}, l_{i}^{+}, i=1,2, \cdots, n$ are constants.

By using the Brouwer's fixed-point theorem, one can easy to prove the existence of the equilibrium point for system(1.2). Assuming that $u^{*}=\left(u_{1}^{*}, u_{2}^{*}, \cdots, u_{n}^{*}\right)^{T}$ is an equilibrium point of (1.2) and by transformation $x(\cdot)=u(\cdot)-u^{*}$, system (1.2) can thus be rewritten as

$$
\begin{gathered}
\dot{x}(t)=-A x(t)+f(W x(t-\tau(t))), \\
x(t)=\varphi(t),-\tau \leq t \leq 0 .
\end{gathered}
$$

Where

$$
f_{j}\left(W_{j} x(t)\right)=g_{j}\left(W_{j}\left(x(t)+u^{*}\right)+J_{j}\right)-g_{j}\left(W_{j} u^{*}+J_{j}\right)
$$

By assumption, it is obvious that

$$
l_{i}^{-} \leq \frac{f_{i}(s)-f_{i}(t)}{s-t} \leq l_{i}^{+}, \forall s \neq t, f_{i}(\mathbf{0})=0, i=1,2, \cdots, n
$$

Then, systems (1.4) admits an equilibrium point $\boldsymbol{x}(\boldsymbol{t}) \equiv \mathbf{0}$ corresponding to the initial condition $\varphi(t) \equiv \mathbf{0 , - \tau \leq t \leq 0}$.

Lemma[14] For any symmetric positive definite matrix $\boldsymbol{M}=\boldsymbol{M}^{T}>\mathbf{0}$, scalar $\boldsymbol{\gamma}>\mathbf{0}$ and vector function $\omega:[0, \gamma] \rightarrow \boldsymbol{R}^{n}$ such that the integrations concerned are well defined, the following integral inequality holds.

$$
\left(\int_{0}^{\gamma} \omega(s) \mathrm{d} s\right)^{T} M\left(\int_{0}^{\gamma} \omega(s) \mathrm{d} s\right) \leq \gamma \int_{0}^{\gamma} \omega^{T}(s) M \omega(s) \mathrm{d} s
$$

\section{MAIN RESULTS}

In this section, the delay fraction ,together with Jensen inequality are employed to obtain our new delay-dependent globally asymptotic stability condition based on a newly constructed Lyapuvov-Krasovskii functional, which contains more information on state variables by taking more states as augment variables.

Theorem For given $\tau, \mu$ and constant matrices $L_{1}=\operatorname{diag}\left(l_{1}^{-}, l_{2}^{-}, \cdots, l_{n}^{-}\right), L_{2}=\operatorname{diag}\left(l_{1}^{+}, l_{2}^{+}, \ldots, l_{n}^{+}\right)$, the origin of system (1.4) is GAS if there exist symmetric positive definite matrices $P, Q_{i} \in \boldsymbol{R}^{n \times n}(i=\mathbf{1 , 2 , 3}), \boldsymbol{R}_{i} \in \boldsymbol{R}^{n \times n}(i=\mathbf{1}, \mathbf{2 , 3 , 4}), Q=\left(\begin{array}{cccc}Q_{11} & Q_{12} & Q_{13} & Q_{14} \\ * & Q_{22} & Q_{23} & Q_{24} \\ * & * & Q_{33} & Q_{34} \\ * & * & * & Q_{44}\end{array}\right) \in \boldsymbol{R}^{4 n \times 4 n}$ nonnegative diagonal matrices $T_{1}=\operatorname{diag}\left(t_{11}, t_{12}, \cdots, t_{1 n}\right), T_{2}=\operatorname{diag}\left(t_{21}, t_{22}, \cdots, t_{2 n}\right)$,

$\Lambda=\operatorname{diag}\left(\lambda_{1}, \lambda_{2}, \cdots, \lambda_{n}\right), \quad \Delta=\operatorname{diag}\left(\delta_{1}, \delta_{2}, \cdots, \delta_{n}\right)$ such that the following LMIs hold.

$$
E_{0}+E_{i}+E_{i j}<0, \quad i=1,2,3,4 ; j=1,2
$$

Where 

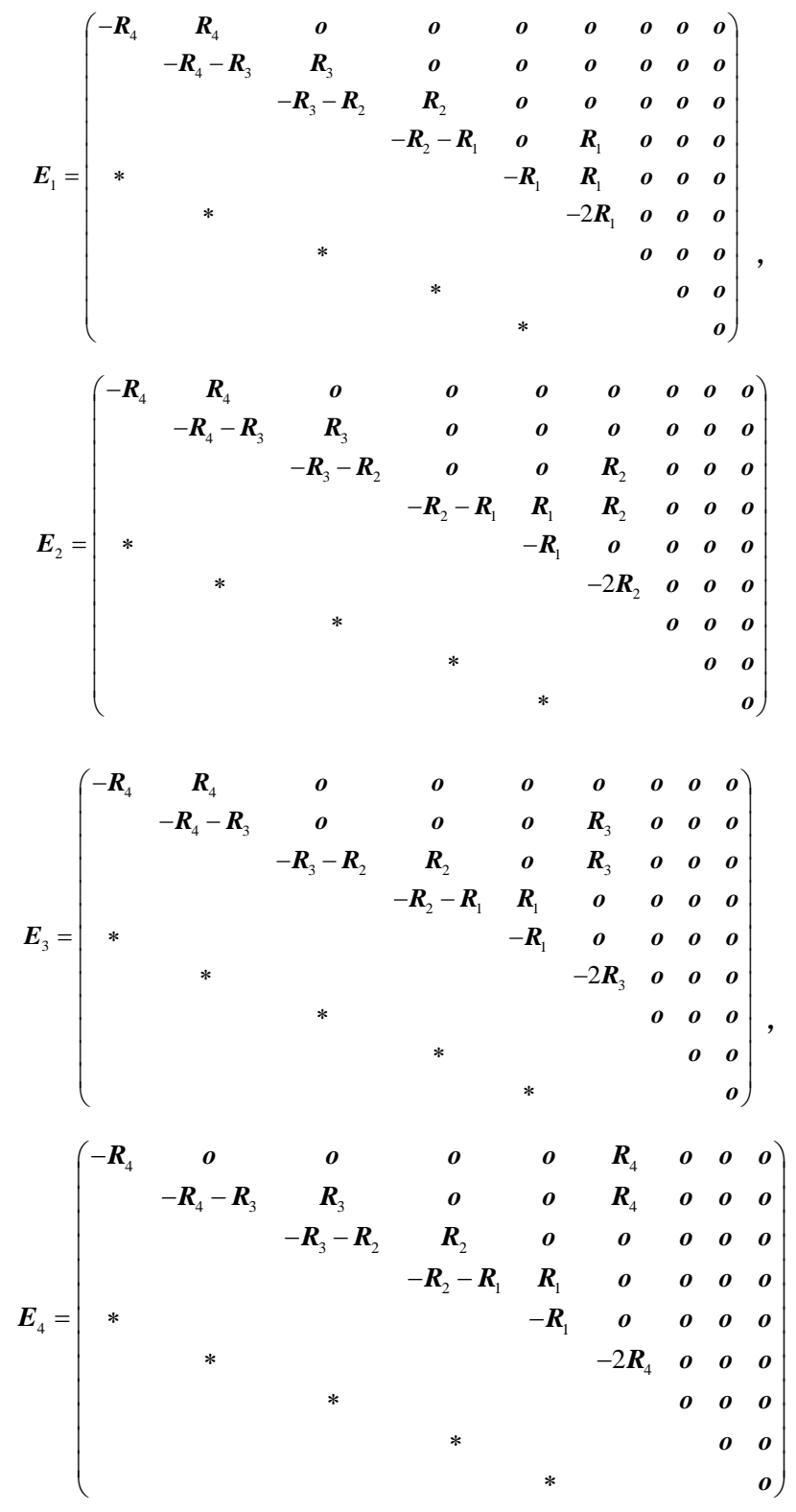

$$
\boldsymbol{E}_{11}=\left(\begin{array}{ccccccccc}
\boldsymbol{o} & \boldsymbol{O} & \boldsymbol{o} & \boldsymbol{o} & \boldsymbol{o} & \boldsymbol{o} & \boldsymbol{o} & \boldsymbol{o} & \boldsymbol{o} \\
& \boldsymbol{o} & \boldsymbol{o} & \boldsymbol{o} & \boldsymbol{o} & \boldsymbol{o} & \boldsymbol{o} & \boldsymbol{o} & \boldsymbol{o} \\
& & \boldsymbol{o} & \boldsymbol{o} & \boldsymbol{o} & \boldsymbol{o} & \boldsymbol{o} & \boldsymbol{o} & \boldsymbol{o} \\
& & & -\boldsymbol{R}_{1} & \boldsymbol{o} & \boldsymbol{R}_{1} & \boldsymbol{o} & \boldsymbol{o} & \boldsymbol{o} \\
& & & \boldsymbol{o} & \boldsymbol{o} & \boldsymbol{o} & \boldsymbol{o} & \boldsymbol{o} & \boldsymbol{o} \\
& & \boldsymbol{R}_{1}^{T} & & -\boldsymbol{R}_{1} & \boldsymbol{o} & \boldsymbol{o} & \boldsymbol{o} \\
& & & & & & \boldsymbol{o} & \boldsymbol{o} & \boldsymbol{o} \\
& & & & & & & \boldsymbol{o} & \boldsymbol{o} \\
& & & * & & & & \boldsymbol{o}
\end{array}\right),
$$
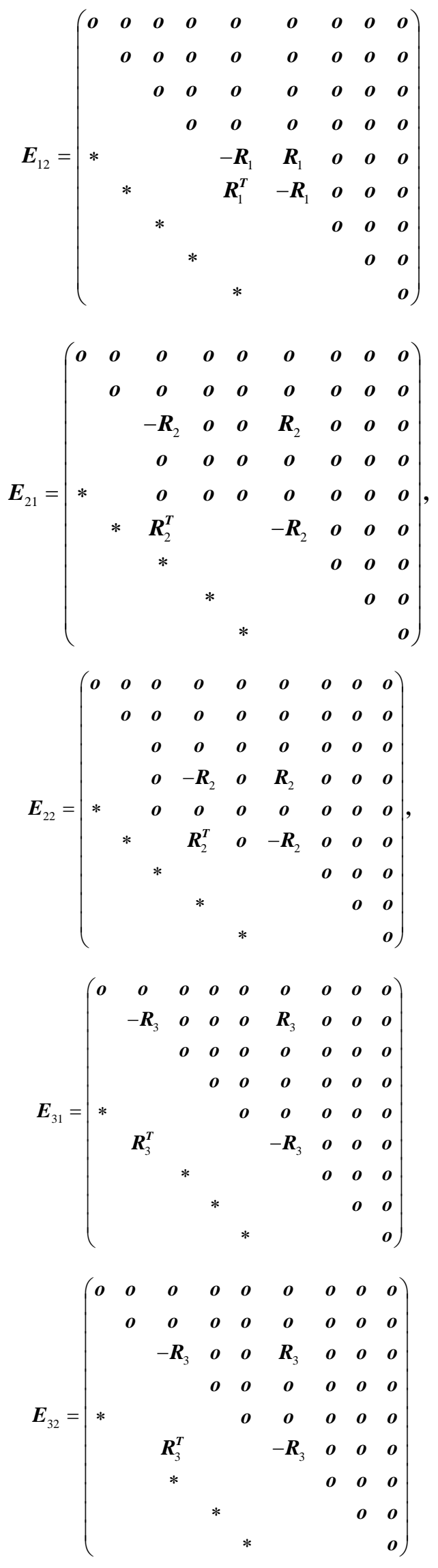


$$
\begin{aligned}
& \boldsymbol{E}_{41}=\left(\begin{array}{ccccccccc}
-\boldsymbol{R}_{4} & \boldsymbol{o} & \boldsymbol{o} & \boldsymbol{o} & \boldsymbol{o} & \boldsymbol{R}_{4} & \boldsymbol{o} & \boldsymbol{o} & \boldsymbol{o} \\
& \boldsymbol{o} & \boldsymbol{o} & \boldsymbol{o} & \boldsymbol{o} & \boldsymbol{o} & \boldsymbol{o} & \boldsymbol{o} & \boldsymbol{o} \\
& & \boldsymbol{o} & \boldsymbol{o} & \boldsymbol{o} & \boldsymbol{o} & \boldsymbol{o} & \boldsymbol{o} & \boldsymbol{o} \\
& & & \boldsymbol{o} & \boldsymbol{o} & \boldsymbol{o} & \boldsymbol{o} & \boldsymbol{o} & \boldsymbol{o} \\
* & & & & \boldsymbol{o} & \boldsymbol{o} & \boldsymbol{o} & \boldsymbol{o} & \boldsymbol{o} \\
\boldsymbol{R}_{4}^{T} & * & & & & -\boldsymbol{R}_{4} & \boldsymbol{o} & \boldsymbol{o} & \boldsymbol{o} \\
& & * & & & & \boldsymbol{o} & \boldsymbol{o} & \boldsymbol{o} \\
& & & * & & & & \boldsymbol{o} & \boldsymbol{o} \\
& & & & * & & & & \boldsymbol{o}
\end{array}\right) \\
& \boldsymbol{E}_{42}=\left(\begin{array}{ccccccccc}
\boldsymbol{o} & \boldsymbol{o} & \boldsymbol{o} & \boldsymbol{o} & \boldsymbol{o} & \boldsymbol{o} & \boldsymbol{o} & \boldsymbol{o} & \boldsymbol{o} \\
& -\boldsymbol{R}_{4} & \boldsymbol{o} & \boldsymbol{o} & \boldsymbol{o} & \boldsymbol{R}_{4} & \boldsymbol{o} & \boldsymbol{o} & \boldsymbol{o} \\
& & \boldsymbol{o} & \boldsymbol{o} & \boldsymbol{o} & \boldsymbol{o} & \boldsymbol{o} & \boldsymbol{o} & \boldsymbol{o} \\
& & & \boldsymbol{o} & \boldsymbol{o} & \boldsymbol{o} & \boldsymbol{o} & \boldsymbol{o} & \boldsymbol{o} \\
* & & & & \boldsymbol{o} & \boldsymbol{o} & \boldsymbol{o} & \boldsymbol{o} & \boldsymbol{o} \\
\boldsymbol{o} & \boldsymbol{R}_{4}^{T} & & & & -\boldsymbol{R}_{4} & \boldsymbol{o} & \boldsymbol{o} & \boldsymbol{o} \\
& & * & & & & \boldsymbol{o} & \boldsymbol{o} & \boldsymbol{o} \\
& & & * & & & & \boldsymbol{o} & \boldsymbol{o} \\
& & & & * & & & & \boldsymbol{o}
\end{array}\right) \\
& E_{0}=\left(\begin{array}{ccccccccc}
E_{11}^{0} & Q_{12} & Q_{13} & Q_{14} & 0 & 0 & 0 & E_{18}^{0} & E_{19}^{0} \\
* & Q_{22}-Q_{11} & Q_{23}-Q_{12} & Q_{24}-Q_{13} & -Q_{14} & 0 & 0 & 0 & 0 \\
* & * & Q_{33}-Q_{22} & Q_{34}-Q_{23} & -Q_{24} & 0 & 0 & 0 & 0 \\
* & * & * & Q_{44}-Q_{33} & -Q_{34} & 0 & 0 & 0 & 0 \\
* & * & * & * & -Q_{44} & 0 & 0 & 0 & 0 \\
* & * & * & * & * & E_{66}^{0} & 0 & 0 & E_{69}^{0} \\
* & * & * & * & * & * & -\left(1-\frac{\mu}{2}\right) Q_{2} & 0 & 0 \\
* & * & * & * & * & * & * & Q_{3}-2 T_{1} & (\Lambda-\Delta) W \\
* & * & * & * & * & * & * & * & -(1-\mu) Q_{3}-2 T_{2}
\end{array}\right) \\
& E_{11}^{0}=-P A-A P+Q_{1}+Q_{2}-2 A^{T} W^{T}\left(L_{2} \Delta-L_{1} \Lambda\right) W+Q_{11}-2 W^{T} L_{1} T_{1} L_{2} W+\left(\frac{\tau}{4}\right)^{2} A^{T} \sum_{i=1}^{4} R_{i} A \\
& E_{18}^{0}=-A^{T} W^{T}(\Lambda-\Delta)+W^{T} T_{1}\left(L_{1}+L_{2}\right) ; E_{19}^{0}=P+W^{T}\left(L_{2} \Delta-L_{1} \Lambda\right) W, \\
& E_{66}^{0}=-(1-\mu) Q_{1}-2 W^{T} L_{1} T_{2} L_{2} W \quad E_{69}^{0}=W^{T} T_{2}\left(L_{1}+L_{2}\right) .
\end{aligned}
$$

Proof. We construct the following Lyapunov functional candidate for system(1.4)

$$
V(x(t))=\sum_{i=1}^{6} V_{i}(x(t))
$$

where

$V_{1}(x(t))=x^{T}(t) P x(t), \quad V_{2}(x(t))=\int_{t-\tau(t)}^{t} x^{T}(s) Q_{1} x(s) \mathrm{d} s+\int_{t-\frac{\tau(t)}{2}}^{t} x^{T}(s) Q_{2} x(s) \mathrm{d} s$,

$$
\begin{gathered}
V_{3}(x(t))=\int_{t-\tau(t)}^{t} f^{T}(W x(s)) Q_{3} f^{T}(W x(s)) \mathrm{d} s \\
V_{4}(x(t))=2 \sum_{i=1}^{n} \lambda_{i} \int_{0}^{W_{i} x(t)}\left[f_{i}(s)-l_{i}^{-} s\right] \mathrm{d} s+2 \sum_{i=1}^{n} \delta_{i} \int_{0}^{W_{i} x(t)}\left[l_{i}^{+} s-f_{i}(s)\right] \mathrm{d} s
\end{gathered}
$$

$V_{5}(x(t))=\int_{t-\frac{\tau}{4}}^{t}\left(x^{T}(s) x^{T}\left(s-\frac{\tau}{4}\right) x^{T}\left(s-\frac{\tau}{2}\right) x^{T}\left(s-\frac{3 \tau}{4}\right)\right)\left(\begin{array}{cccc}Q_{11} & Q_{12} & Q_{13} & Q_{14} \\ * & Q_{22} & Q_{23} & Q_{24} \\ * & * & Q_{33} & Q_{34} \\ * & * & * & Q_{44}\end{array}\right)\left(\begin{array}{c}x(s) \\ x\left(s-\frac{\tau}{4}\right) \\ x\left(s-\frac{\tau}{2}\right) \\ x\left(s-\frac{3 \tau}{4}\right)\end{array}\right) \mathrm{d} s$,
$V_{6}(x(t))=\frac{\tau}{4} \int_{-\tau}^{-\frac{3 \tau}{4}} \int_{t+\theta}^{t} \dot{x}^{T}(s) R_{1} \dot{x}(s) \mathrm{d} s \mathrm{~d} \theta+\frac{\tau}{4} \int_{-\frac{3 \tau}{4}}^{-\frac{\tau}{2}} \int_{t+\theta}^{t} \dot{x}^{T}(s) R_{2} \dot{x}(s) \mathrm{d} s \mathrm{~d} \theta$

$+\frac{\tau}{4} \int_{-\frac{\tau}{2}}^{-\frac{\tau}{4}} \int_{t+\theta}^{t} \dot{x}^{T}(s) R_{3} \dot{x}(s) \mathrm{d} s \mathrm{~d} \theta+\frac{\tau}{4} \int_{-\frac{\tau}{4}}^{0} \int_{t+\theta}^{t} \dot{x}^{T}(s) R_{4} \dot{x}(s) \mathrm{d} s \mathrm{~d} \theta$

where $w_{i}$ in $V_{4}(x(t))$ denotes the $i$ th row of matrix $w$.

In what follows, we compute the time derivative of $V(x(t))$ along the trajectory of system(1.4).

$$
\begin{aligned}
\dot{V}_{1}(x(t))= & 2 x^{T}(t) P \dot{x}(t)=2 x^{T}(t) P[-A x(t)+f(W x(t-\tau(t)))] \\
\dot{V}_{2}(x(t)) & \leq x^{T}(t)\left(Q_{1}+Q_{2}\right) x(t)-(1-\mu) x^{T}(t-\tau(t)) Q_{1} x(t-\tau(t)) \\
& -\left(1-\frac{\mu}{2}\right) x^{T}\left(t-\frac{\tau(t)}{2}\right) Q_{2} x\left(t-\frac{\tau(t)}{2}\right)
\end{aligned}
$$

$\dot{V}_{3}(x(t)) \leq f^{T}(W x(t)) Q_{3} f(W x(t))-(1-\mu) f^{T}(W x(t-\tau(t))) Q_{3} f^{T}(W x(t-\tau(t)))$ the above 2 inequalities hold for $i(t) \leq \mu$.

By Lemma1, we have

$$
\begin{gathered}
\dot{V}_{4}(x(t))=2 \sum_{i=1}^{n} \lambda_{i}\left[f_{i}\left(W_{i} x(t)\right)-l_{i}^{-} W_{i} x(t)\right] \cdot W_{i} \dot{x}(t)+2 \sum_{i=1}^{n} \delta_{i}\left[l_{i}^{+} W_{i} x(t)-f_{i}\left(W_{i} x(t)\right)\right] \cdot W_{i} \dot{x}(t) \\
=2 \dot{x}^{T}(t) W^{T}\left(L_{2} \Delta-L_{1} \Lambda\right) W x(t)+2 f^{T}(W x(t))(\Lambda-\Delta) W \dot{x}(t) \\
=2\left(-x^{T}(t) A^{T}+f^{T}(W x(t-\tau(t)))\right) W^{T}\left(L_{2} \Delta-L_{1} \Lambda\right) W x(t) \\
\quad+2 f^{T}(W x(t))(\Lambda-\Delta) W(-A x(t)+f(W x(t-\tau(t))))
\end{gathered}
$$

$$
\dot{V}_{5}(x(t))=\left(x^{T}(t), x^{T}\left(t-\frac{\tau}{4}\right), x^{T}\left(t-\frac{\tau}{2}\right), x^{T}\left(t-\frac{3 \tau}{4}\right)\right)\left(\begin{array}{cccc}
Q_{11} & Q_{12} & Q_{13} & Q_{14} \\
* & Q_{22} & Q_{23} & Q_{24} \\
* & * & Q_{33} & Q_{34} \\
* & * & * & Q_{44}
\end{array}\right)\left(\begin{array}{c}
x(t) \\
x\left(t-\frac{\tau}{4}\right) \\
x\left(t-\frac{\tau}{2}\right) \\
x\left(t-\frac{3 \tau}{4}\right)
\end{array}\right)
$$

$$
-\left(x^{T}\left(t-\frac{\tau}{4}\right) x^{T}\left(t-\frac{\tau}{2}\right) x^{T}\left(t-\frac{3 \tau}{4}\right) x^{T}(t-\tau)\right)\left(\begin{array}{cccc}
Q_{11} & Q_{12} & Q_{13} & Q_{14} \\
* & Q_{22} & Q_{23} & Q_{24} \\
* & * & Q_{33} & Q_{34} \\
* & * & * & Q_{44}
\end{array}\right)\left(\begin{array}{c}
x\left(t-\frac{\tau}{4}\right) \\
x\left(t-\frac{\tau}{2}\right) \\
x\left(-\frac{3 \tau}{4} t\right) \\
x(t-\tau)
\end{array}\right)
$$

$\dot{V}_{6}(x(t))=\left(\frac{\tau}{4}\right)^{2} \dot{x}^{T}(t)\left(R_{1}+R_{2}+R_{3}+R_{4}\right) \dot{x}(t)-\frac{\tau}{4} \int_{t-\tau}^{t-\frac{3 \tau}{4}} \dot{x}^{T}(s) R_{1} \dot{x}(s) \mathrm{d} s$

$-\frac{\tau}{4} \int_{t-\frac{\pi \tau}{4}}^{t-\frac{\tau}{2}} \dot{x}^{T}(s) R_{2} \dot{x}(s) \mathrm{d} s-\frac{\tau}{4} \int_{t-\frac{\tau}{2}}^{t-\frac{\tau}{4}} \dot{x}^{T}(s) R_{3} \dot{x}(s) \mathrm{d} s-\frac{\tau}{4} \int_{t-\frac{\tau}{4}}^{t} \dot{x}^{T}(s) R_{4} \dot{x}(s) \mathrm{d} s$. 
In order to estimate an elaborate upper bound of $\dot{V}_{6}(x(t))$, different cases are considered as follows. When $\frac{3}{4} \tau \leq \tau(t) \leq \tau$, we have

$$
\begin{gathered}
-\frac{\tau}{4} \int_{t-\tau}^{t-\frac{3 \tau}{4}} \dot{x}^{T}(s) R_{1} \dot{x}(s) \mathrm{d} s=-\frac{\tau}{4} \int_{t-\tau}^{t-\tau(t)} \dot{\boldsymbol{x}}^{T}(s) R_{1} \dot{x}(s) \mathrm{d} s-\frac{\tau}{4} \int_{t-\tau(t)}^{t-\frac{3 \tau}{\tau}} \dot{\boldsymbol{x}}^{T}(s) R_{1} \dot{x}(s) \mathrm{d} s \\
\text { Define } \alpha_{1}=\frac{4 \tau-4 \tau(t)}{\tau}, \text { then }
\end{gathered}
$$$$
-\frac{\tau}{4} \int_{t-\tau}^{t-\tau(t)} \dot{x}^{T}(s) R_{1} \dot{x}(s) \mathrm{ds}=-(\tau-\tau(t)) \int_{t-\tau}^{t-\tau(t)} \dot{x}^{T}(s) R_{1} \dot{x}(s) \mathrm{ds}-\left(\tau(t)-\frac{3 \tau}{4}\right) \int_{t-\tau}^{t-\tau(t)} \dot{x}^{T}(s) R_{1} \dot{x}(s) \mathrm{ds}
$$$$
\leq-(\tau-\tau(t)) \int_{t-\tau}^{t-\tau(t)} \dot{x}^{T}(s) R_{1} \dot{x}(s) \mathrm{d} s-\left(1-\alpha_{1}\right)(\tau-\tau(t)) \int_{t-\tau}^{t-\tau(t)} \dot{x}^{T}(s) R_{1} \dot{x}(s) \mathrm{d} s
$$

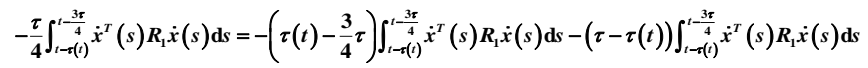$$
\leq-\left(\tau(t)-\frac{3}{4} \tau\right) \int_{t-\tau(t)}^{t-\frac{3 \tau}{4}} \dot{x}^{T}(s) R_{1} \dot{x}(s) \mathrm{d} s-\alpha_{1}\left(\tau(t)-\frac{3}{4} \tau\right) \int_{t-\tau(t)}^{t-\frac{3 \tau}{4}} \dot{x}^{T}(s) R_{1} \dot{x}(s) \mathrm{d} s
$$

Therefore, Lemma2 gives that

$$
\begin{gathered}
-\frac{\tau}{4} \int_{t-\tau}^{t-\frac{3 \tau}{4}} \dot{x}^{T}(s) R_{1} \dot{x}(s) \mathrm{d} s \leq-\left[x^{T}(t-\tau(t))-x^{T}(t-\tau)\right] R_{1}[x(t-\tau(t))-x(t-\tau)] \\
-\left[x^{T}\left(t-\frac{3}{4} \tau\right)-x^{T}(t-\tau(t))\right] R_{1}\left[x\left(t-\frac{3}{4} \tau\right)-x(t-\tau(t))\right] \\
-\alpha_{1}\left[x^{T}\left(t-\frac{3}{4} \tau\right)-x^{T}(t-\tau(t))\right] R_{1}\left[x\left(t-\frac{3}{4} \tau\right)-x(t-\tau(t))\right] \\
-\left(1-\alpha_{1}\right)\left[x^{T}(t-\tau(t))-x^{T}(t-\tau)\right] R_{1}[x(t-\tau(t))-x(t-\tau)] .(14)
\end{gathered}
$$

and

$$
\begin{aligned}
& -\frac{\tau}{4} \int_{t \frac{3 \pi}{4}}^{t \frac{\tau}{2}} \dot{x}^{T}(s) R_{2} \dot{x}(s) \mathrm{d} s \leq-\left[x^{T}\left(t-\frac{\tau}{2}\right)-x^{T}\left(t-\frac{3 \tau}{4}\right)\right] R_{2}\left[x\left(t-\frac{\tau}{2}\right)-x\left(t-\frac{3 \tau}{4}\right)\right] . \\
& -\frac{\tau}{4} \int_{t-\frac{\tau}{2}}^{t} \int^{\frac{\tau}{4}} \dot{x}^{T}(s) R_{3} \dot{x}(s) \mathrm{d} s \leq-\left[x^{T}\left(t-\frac{\tau}{4}\right)-x^{T}\left(t-\frac{\tau}{2}\right)\right] R_{3}\left[x\left(t-\frac{\tau}{4}\right)-x\left(t-\frac{\tau}{2}\right)\right] . \\
& -\frac{\tau}{4} \int_{t \frac{\tau}{4}}^{t} \dot{x}^{T}(s) R_{4} \dot{x}(s) \mathrm{d} s \leq-\left[x^{T}(t)-x^{T}\left(t-\frac{\tau}{4}\right)\right] R_{4}\left[x(t)-x\left(t-\frac{\tau}{4}\right)\right] .
\end{aligned}
$$

Moreover, according to (1.5), there exists nonnegative diagonal matrices $T_{1}=\operatorname{diag}\left(t_{11}, t_{12}, \cdots, t_{t_{11}}\right), T_{2}=\operatorname{diag}\left(t_{21}, t_{22}, \cdots, t_{t_{2 n}}\right)$, such that the following inequalities hold,

$$
\begin{gathered}
2 x^{T}(t) W^{T} T_{1}\left(L_{1}+L_{2}\right) f(W x(t))-2 x^{T}(t) W^{T} L_{T_{1}} L_{2} W x(t)-2 f^{T}(W x(t)) T_{1} f(W x(t)) \geq 0 \\
2 x^{T}(t-\tau(t)) W^{T} T_{2}\left(L_{1}+L_{2}\right) f(W x(t-\tau(t)))-2 x^{T}(t-\tau(t)) W^{T} L_{1} T_{2} L_{2} W x(t-\tau(t)) \\
-2 f^{T}(W x(t-\tau(t))) T_{2} f(W x(t-\tau(t))) \geq 0
\end{gathered}
$$

From (1.8) (1.19),we have

$$
\begin{gathered}
\dot{V}(x(t)) \leq \dot{V}(x(t))+2 x^{T}(t) W^{T} T_{1}\left(L_{1}+L_{2}\right) f(W x(t))-2 x^{T}(t) W^{T} L_{1} T_{1} L_{2} W x(t) \\
-2 f^{T}(W x(t)) T_{1} f(W x(t))+2 x^{T}(t-\tau(t)) W^{T} T_{2}\left(L_{1}+L_{2}\right) f(W x(t-\tau(t))) \\
-2 x^{T}(t-\tau(t)) W^{T} L_{1} L_{2} L_{2} W x(t-\tau(t))-2 f^{T}(W x(t-\tau(t))) T_{2} f(W x(t-\tau(t))) \\
\quad \leq \xi^{T}(t)\left[E_{0}+E_{1}+\alpha_{1} E_{11}+\left(1-\alpha_{1}\right) E_{12}\right] \xi(t)
\end{gathered}
$$

where

$$
\xi^{r}(t)=\left(x^{r}(t), x^{r}\left(t-\frac{\tau}{4}\right), x^{r}\left(t-\frac{\tau}{2}\right), x^{r}\left(t-\frac{3 \tau}{4}\right), x^{r}(t-\tau), x^{r}(t-\tau(t)), x^{r}\left(t-\frac{\tau(t)}{2}\right), f^{r}(W x(t)), f^{\tau}(W x(t-\tau(t)))\right)
$$
$E_{0}, E_{1}, E_{11}, E_{12}$ are given as above.

Note that $0 \leq \alpha_{1} \leq \mathbf{1}$, the above left hand of (1.20) induces a convex combination of matrices $E_{11}$ and $E_{12}$, which are negative definite only if the vertices are. According to (1.18), (1.19), (1.9)

and (1.7),together with S-procedure. One can easily get there exists a positive constant $\varepsilon$ such that

$$
E_{0}+E_{1}+\alpha_{1} E_{11}+\left(1-\alpha_{1}\right) E_{12}<\operatorname{diag}(-\varepsilon I, o, o, o, o, o, o, o, o),
$$

By pre- and post-multiplying the above inequality with $\xi^{T}(t), \xi(t)$ respectively, one can easily achieve

$$
\dot{V}(x(t)) \leq \xi^{T}(t)\left[E_{0}+E_{1}+\alpha_{1} E_{11}+\left(1-\alpha_{1}\right) E_{12}\right] \xi(t)<-\varepsilon\|x(t)\|^{2}
$$

Which implies the origin of system(1.4) is globally asymptotically stable for the case of $\frac{3}{4} \tau \leq \tau(t) \leq \tau$.

Similarly, when $\frac{1}{2} \tau \leq \tau(t) \leq \frac{3}{4} \tau, \frac{1}{4} \tau \leq \tau(t) \leq \frac{1}{2} \tau, 0 \leq \tau(t) \leq \frac{1}{2} \tau$, respectively, one can get

$$
\begin{gathered}
\dot{V}(x(t)) \leq \xi^{T}(t)\left[\alpha_{2} E_{21}+\left(1-\alpha_{2}\right) E_{22}\right] \xi(t), \\
\dot{V}(x(t)) \leq \xi^{T}(t)\left[\alpha_{3} E_{31}+\left(1-\alpha_{3}\right) E_{32}\right] \xi(t), \\
\dot{V}(x(t)) \leq \xi^{T}(t)\left[\alpha_{4} E_{41}+\left(1-\alpha_{4}\right) E_{42}\right] \xi(t),
\end{gathered}
$$

By the same way to the first case.

Where $\alpha_{2}=\frac{3 \tau-4 \tau(t)}{\tau}, \alpha_{3}=\frac{2 \tau-4 \tau(t)}{\tau} \quad \alpha_{4}=\frac{\tau-4 \tau(t)}{\tau}$, respectively.

For simplicity, the details were omitted here, and we complete the proof.

Remark1 In many literatures, the time-varying delay $\tau(t)$ must be differentiable and $\dot{i}(t)<1$. But in this paper, the constraint $i(t)<1$ can be relaxed because of the positive definiteness of matrices $Q_{1}, Q_{2}$ and $Q_{3}$, then the constraint 
condition $\dot{i}(t)<1$ can be relaxed as $\dot{i}(t)<\mu$, where $\mu$ is not necessarily smaller than 1 .

$$
\begin{gathered}
\text { Remark2 By taking states } \\
x\left(t-\frac{\tau}{4}\right), x\left(t-\frac{\tau}{2}\right), x\left(t-\frac{3 \tau}{4}\right), x(t-\tau), x(t-\tau(t)),
\end{gathered}
$$
this paper utilizes more information on state variables, this is an efficient method to derive a less conservative stability result.

Remark3 The delay interval $[0, \tau]$ is divided into 4 segments, each segment has a different positive Lyapunov matrix, and matrix used to describe the relation between different segments is also set, which has potential to yield less conservative stability condition.

Remark4 To reduce the conservation, an elaborate estimation of the upper bound of the integral item in $\dot{V}_{6}(x(t))$ is made. For example, when $\frac{3}{4} \tau \leq \tau(t) \leq \tau,-\frac{\tau}{4} \int_{t-\tau}^{t-\frac{3 \tau}{4}} \dot{x}^{T}(s) R_{1} \dot{x}(s) \mathrm{d} s$ is not simply up bounded as $-\frac{\tau}{4} \int_{t-\tau}^{t \tau(t)} \dot{\boldsymbol{x}}^{T}(s) \boldsymbol{R}_{1} \dot{x}(s) \mathrm{ds}$, but $-\frac{\tau}{4} \int_{t-\tau(t)}^{t-\frac{3 \tau}{4}} \dot{\boldsymbol{x}}^{T}(s) \boldsymbol{R}_{1} \dot{x}(s) \mathrm{d} s$ is also considered. Especially, the latter is not over bounded as $-\left(\tau(t)-\frac{3}{4} \tau\right) \int_{t-\tau(t)}^{t-\frac{3 \tau}{4}} \dot{x}^{T}(s) R_{1} \dot{x}(s) \mathrm{ds}$, but rather $-(\tau-\tau(t)) \int_{t-\tau(t)}^{t-\frac{3 \tau}{4}} \dot{x}^{T}(s) R_{1} \dot{x}(s) \mathrm{d}$ is taken into account. The same way is conducted on the other 3 integral items in $\dot{V}_{6}(x(t))$.

Remark5 Be different with the approach as in [8], the free-weighting matrix was not introduced in this paper. As it was pointed that the free-weighting matrix may has no any contribution to a less conservative stability condition, while the computation complex is burdened.

It is worth noting that theorem 1 does not necessarily require $\mu<1$, that means it can be applied to fast time-varying delays as well as slow ones, only if $\mu$ is known. While in practice, $\mu$ is unknown or $\tau(t)$ is not differentiable, by setting $Q_{1}=Q_{2}=Q_{3}=\mathbf{0}$, a delay-rate-independent criterion for fast timevarying delays can be obtained easily as follows.

Corollary For given $\tau$ and constant matrices $L_{1}=\operatorname{diag}\left(l_{1}^{-}, l_{2}^{-}, \cdots, l_{n}^{-}\right), L_{2}=\operatorname{diag}\left(l_{1}^{+}, l_{2}^{+}, \cdots, l_{n}^{+}\right)$, the origin of system (1.4) is GAS if there exist symmetric positive definite matrices $P, R_{i}(i=\mathbf{1 , 2 , 3 , 4}), Q=\left(\begin{array}{cccc}Q_{11} & Q_{12} & Q_{13} & Q_{14} \\ * & Q_{22} & Q_{23} & Q_{24} \\ * & * & Q_{33} & Q_{34} \\ * & * & * & Q_{44}\end{array}\right)$, nonnegative diagonal matrices $T_{1}=\operatorname{diag}\left(t_{11}, t_{12}, \cdots, t_{1 n}\right), T_{2}=\operatorname{diag}\left(t_{21}, t_{22}, \cdots, t_{2 n}\right)$,

$\Lambda=\operatorname{diag}\left(\lambda_{1}, \lambda_{2}, \cdots, \lambda_{n}\right), \quad \Delta=\operatorname{diag}\left(\delta_{1}, \delta_{2}, \cdots, \delta_{n}\right)$ such that the following LMIs hold.

$$
\tilde{E}^{0}+E_{i}+E_{i j}<0, \quad i=1,2,3,4 ; j=1,2
$$

where

$\tilde{E}_{11}^{0}=-P A-A P-2 A^{T} W^{T}\left(L_{2} \Delta-L_{1} \Lambda\right) W+Q_{11}-2 W^{T} L_{1} T_{1} L_{2} W+\left(\frac{\tau}{4}\right)^{2} A^{T} \sum_{i=1}^{4} R_{i} A$,

$\tilde{E}_{66}^{0}=-2 W^{T} L_{1} T_{2} L_{2} W, \tilde{E}_{88}^{0}=-2 T_{1}, \tilde{E}_{99}^{0}=-2 T_{2} ; \tilde{E}_{i j}^{0}=E_{i j}^{0}$, for other $i, j$ as defined in theorem1.

\section{CONCLUSION}

By a newly constructed Lyapunov-Krasovskii functional, together with the delay fractioning method and convex combination technique, an improved delay-dependent globally asymptotical stability sufficient condition has been obtained. The condition is given in terms of LMIs and thus can be readily checked through the standard numerical software. Example shows significantly that delay fractioning method and the elaborate bounding technique convex combination can reduce the conservatism effectively.

\section{REFERENCES}

[1] L.Chua,L.Yang, Cellular neural networks:applications, IEEE Trans.Circuits Syst.I 35(1998)1273-1290.

[2] N.Ozcan,S.Arik, Global robust stability analysis of neural networks with multiple time delays, IEEE Trans.Circuits Syst, I 35(1)(2006)166-176.

[3] Z.Xu,H.Qiao,J.Peng,J.Cao. A comparative study of two modeling approaches in neural networks, Neural networks, 17(2004)73-85.

[4] X.Liang,J.Wang. A recurrent neural networks for nonlinear optimization with a continuously differentiable objective function and bound constraints. IEEE Transactions on neural networks, 11(6) (2000)12511261.

[5] S.Hu,J.Wang. Global stability of a class of continuous-time recurrent neural networks, IEEE transactions on circuits and systems-I: fundermental theory and applications, 49(9)(2002)1334-1347.

[6] S.Xu,J.Lam,D.W.C.Ho,Y.Zou, Global robust exponential stability analysis for interval recurrent neural networks, Phys.Lett.A 325(2004)124-133.

[7] J.Liang,J.Cao, A based-on LMI stability criterion for delayed recurrent neural networks, Chaos,Solitons Fractals, 28(2006)154-160.

[8] C.-Y.Lu,T.-J.Su,S.-C.Huang, Delay-dependent stability analysis for recurrent neural networks with time-varying delay, IET Control Theory Appl, 2(8)(2008)736-742.

[9] ]H.Shao, Less conservative delay-dependent stability criteria for neural neyworks with time-varying delays, Neurocomputing, 73(2010)15281532.

[10] P.Li,J.Cao, Stability in static delayed neural networks :A nonlinear measure approach, Neurocomputing, 69(2006)1776-1781.

[11] X.Jiang,Q.Han,S.liu,A.Xue, A new $H_{\infty}$ stabilization criterion for networked control systems, IEEE Trans. Auto Control, 53(4)(2008)1025-1032.

[12] D.Peaucelle, F.Gouaisbaut, Discussion on:"parameter-dependent Lyapunov function approach to stability analysis and design for uncertain systems with time-varying delay". Eur.J.Control, 11(1)(2005)69-79.

[13] X.Zhu,Y.Wang, Delay-dependent exponential stability for neural networks with discrete and distributed time-varying dalays, Phys.Lett.A, 373(2009)4066-4072.

[14] K.Gu, An integral inequality in the stability problem of time delay systems, in: proceedings of 39th IEEE conference on decision control, 2000,2805-2810.

[15] T.Li,L.Guo,C.Lin, Further results on delay-dependent stability criteria for neural networks with time-varying delays, IEEE Trans. Neural networks 19(4)(2008)726-730.

[16] Q.Zhang,X.Wei,J.Xu, Delay-dependent global stability results for delayed Hopfeild neural networks, Chaos, sollitons Fractals 34(2007)662-668.

[17] S.Xu,J.Lam,D.W.C.Ho,Y.Zhou, Novel Global asymptotic stability criteria for delayed cellular neural networks, IEEE Trans.Circuits Syst.II 52(2005)349-353.

[18] S.Xu,J.Lam,D.W.C.Ho, A new LMI condition for delayed dependent asymptotic stability of delayed Hopfeild neural networks, IEEE Trans.Circuits Syst.II 52(3)(2006)230-234. 
[19] H.Cho,J.Park, Novel delay-dependent robust stability criterion of delayed cellular neural networks, Chaos, sollitons Fractals 32(2007)1194-1200.

[20] Y.He,G.Liu,D.Rees, New delay-dependent stability criteria for neural networks with time-varying delay, IEEE Trans. Neural Networks18(1)(2007)310-314

[21] W.Chen,W.zeng, Global asymptotic stability of a class of neural networks with distributed delays, IEEE Trans.Circuits Syst.I 53(3)(2006)644-652.

[22] X.Liao,Q.Liu,W.Zhang, Delay-dependent asymptotic stability for neural networks with distributed delays, Nonlinear Anal.: Real World Appl.7(2006)1178-1192.

[23] Y.Yang,J.D.Cao, solving quadratic programming problems by delayed projection neural network, IEEE Trans.Neural Netw.17(2006)1630-1634.

[24] F.Li,delyed Lagrangian neural networks for solving convex programming problems, Neurocomputing.73(2010)2266-2273. 